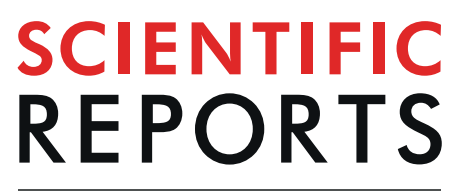

natureresearch

\title{
Early postoperative weight gain is associated with increased risk of graft failure in living donor liver transplant recipients
}

Hye-Won Jeong $\mathbb{1}^{1}$, Kyeo-Woon Jung ${ }^{2}$, Seon-Ok Kim ${ }^{3}$, Hye-Mee Kwon $\mathbb{D}^{2}$, Young-Jin Moon ${ }^{2}$, In-Gu Jun $\mathbb{D}^{2}$, Jun-Gol Song $\mathbb{1}^{2 *}$ \& Gyu-Sam Hwang $\mathbb{C}^{2}$

Fluid overload (FO) has been shown to adversely affect multiple organs and survival in critically ill patients. Liver transplantation (LT) carries the risk of massive transfusion, which frequently results in FO. We investigated the association of postoperative weight gain with graft failure, early allograft dysfunction (EAD), and overall mortality in LT. 1833 living donor LT (LDLT) recipients were retrospectively analysed. Patients were divided into 2 groups according to postoperative weight gain ( $<3 \%$ group $[n=1391]$ and $\geq 3 \%$ group $[n=442]$ ) by using maximally selected log-rank statistics for graft failure. Multivariate Cox and logistic regression analyses were performed. The $\geq 3 \%$ group was associated with graft failure (adjusted $\mathrm{HR}[\mathrm{aHR}], 1.763 ; 95 \% \mathrm{Cl}, 1.248-2.490 ; P=0.001$ ). When postoperative weight change was used as a continuous variable, the aHR for each $1 \%$ increase in postoperative weight was $1.045(95 \% \mathrm{Cl}, 1.009-1.082 ; P=0.015)$. In addition, the $\geq 3 \%$ group was associated with EAD (adjusted OR [aOR], $1.553 ; 95 \% \mathrm{Cl}, 1.024-2.356 ; P=0.038$ ) and overall mortality (aHR, $1.731 ; 95 \% \mathrm{Cl}, 1.182-2.535 ; P=0.005)$. In conclusion, postoperative weight gain may be independently associated with increased risk of graft failure, EAD, and mortality in LDLT recipients.

Liver transplantation (LT) carries the risk of massive transfusion, which frequently results in fluid overload (FO). There is growing evidence that FO can be harmful to critically ill patients or those undergoing major abdominal surgery $^{1-6}$, with the adverse effects persisting even after the fluid status has improved ${ }^{7}$. LT recipients may be particularly vulnerable to FO owing to their fragile cardiopulmonary status, resulting in increased complications and prolonged stay in the intensive care unit (ICU) ${ }^{8-10}$.

Graft survival after LT has been found to be associated with various factors, including donor and recipient age, model for end-stage liver disease (MELD) score, and ischaemic time ${ }^{11,12}$. Meanwhile, FO has been reported to have adverse impacts on multiple organ systems $s^{13,14}$. FO may cause endothelial dysfunction and bacterial or endotoxin translocation, leading to a state of systemic inflammation ${ }^{15,16}$, which may result in decompensation and multiorgan failure in advanced liver cirrhosis patients ${ }^{17}$. Systemic inflammation may also provoke hepatic angiogenesis of liver transplants, leading to fibrosis and cirrhotic remodelling that ultimately result in decreased graft survival in LT recipients ${ }^{18-20}$.

Despite the link between FO, inflammation, and organ or graft dysfunction, the impact of perioperative FO on graft function in LT recipients has not been well elucidated. Therefore, we investigated the relationship between early postoperative weight gain and the occurrence of graft failure in living donor LT (LDLT) recipients. We also evaluated the occurrence of early allograft dysfunction (EAD), as well as overall mortality.

\section{Methods}

This retrospective observational study was approved by the Institutional Review Board of Asan Medical Centre (Seoul, South Korea), which did not require written informed consent due to the retrospective design of the study. All methods were performed in accordance with the relevant guidelines and regulations.

${ }^{1}$ Department of Anaesthesiology and Pain Medicine, International St. Mary's Hospital, Catholic Kwandong University College of Medicine, Incheon, Korea. ${ }^{2}$ Department of Anaesthesiology and Pain Medicine, Laboratory for Cardiovascular Dynamics, Asan Medical Centre, University of Ulsan College of Medicine, Seoul, Korea. ${ }^{3}$ Department of Clinical Epidemiology and Biostatistics, Asan Medical Centre, University of Ulsan College of Medicine, Seoul, Korea. *email: jungol.song@amc.seoul.kr 
Patients. All patients who underwent LT at our institution, from January 2008 to September 2015, were enrolled. The Hospital Based Organ Procurement Organization designated by law procured living or deceased liver grafts, and none of the grafts were obtained from executed prisoners. We excluded patients (1) who were $<18$ years old, (2) who underwent orthotopic LT, and (3) whose preoperative serum creatinine level was $>1.5 \mathrm{mg} /$ $\mathrm{dL}$. The clinical data of the remaining patients, including demographic data, liver disease, perioperative laboratory data, donor data, intraoperative data containing anaesthetic management and surgical data, and postoperative morbidity and mortality were obtained from the computerized databases (electronic medical records system) of our institution, and analysed.

Intraoperative management. Anaesthesia and haemodynamic monitoring were performed according to our institutional standard ${ }^{21}$. Briefly, we maintained anaesthesia with sevoflurane or desflurane, a mixture of $50 \%$ $\mathrm{O}_{2}$ and $50 \%$ air, and a continuous infusion of fentanyl. Arterial pressure was monitored using radial and femoral arterial catheters. For advanced haemodynamic monitoring, a pulmonary arterial catheter was inserted and connected to a Vigilance device (Vigilance II $^{\circledR}$, Edwards Lifesciences, Irvine, CA, USA). According to the patient's blood pressure and haemodynamics, the attending anaesthesiologist administered either fluid or vasoactive drugs. In cases of low systemic vascular resistance, a continuous infusion of norepinephrine or vasopressin was used to maintain the mean arterial blood pressure either above the preoperative level or $65 \mathrm{mmHg}$. Intraoperative transfusions of packed red blood cells (pRBCs), fresh frozen plasma (FFP), cryoprecipitate, and apheresis platelets were based on institutional standards adopted from clinical and rotational thromboelastometry guidelines, with the aim of maintaining prothrombin time (international normalized ratio [INR]) $<2.0$, fibrinogen $>100 \mathrm{mg} / \mathrm{dL}$, and platelet counts $>30,000 / \mu \mathrm{L}$.

Postoperative management. In the postoperative period, our institution's goal of fluid management is to maintain near-zero fluid balance to minimize weight changes in the ICU ${ }^{22,23}$. Because FO may lead to graft congestion, especially in LDLT recipients, LT recipients are weighed every day and their fluid balance is adjusted accordingly. Patients with massive ascites before surgery are managed with a more liberal fluid management strategy to prevent renal dysfunction such as acute kidney injury (AKI).

Postoperative weight change. Postoperative weight change (\%) was calculated using the following formula: ([body weight on the morning after surgery - body weight on the morning before surgery]/body weight on the morning before surgery) $\times 100$.

Inflammatory parameters. Serum C-reactive protein (CRP) and neutrophil-to-lymphocyte ratio (NLR) were routinely measured every morning from the preoperative day to postoperative day (POD) 7. Serum CRP level was measured using a latex-enhanced immunoturbidimetric assay on the Cobas 8000 analyser (Roche Diagnostics, Mannheim, Germany), with the CRPL3 reagent (Roche). The diagnostic range of this assay is 0.03 to $35 \mathrm{mg} / \mathrm{dL}$, with a reference value of $<0.6 \mathrm{mg} / \mathrm{dL}$. NLR is a marker of systemic inflammation that can be easily obtained from the differential white blood cell count. The NLR was obtained by dividing absolute neutrophil counts by absolute lymphocyte counts.

Outcome variables. The main outcome of this study was the occurrence of graft failure after LDLT. Graft failure was defined as the earlier of re-transplantation or death from any cause. Other outcomes included EAD, overall mortality, and lengths of ICU and hospital stay. EAD was defined by the presence of one or both of the following variables: bilirubin $>10 \mathrm{mg} / \mathrm{dL}$ or prothrombin time (INR) $>1.6$ on POD $7^{24}$. Graft failure and overall mortality were assessed using electronic medical records and a registry that was regularly updated by the Asan Organ Transplantation Centre. In addition, the prevalence of pulmonary complications or AKI after LDLT was assessed. According to the Kidney Disease: Improving Global Outcomes (KDIGO) criteria, AKI was defined as an increase in serum creatinine by $\geq 0.3 \mathrm{mg} / \mathrm{dL}$ within 48 hours or an increase in serum creatinine to $\geq 1.5$ times baseline, which is known or presumed to have occurred within the prior 7 days $^{25}$.

Statistical analysis. Continuous variables are reported as mean \pm standard deviation or median (interquartile range $[\mathrm{IQR}]$ ), and categorical variables as frequency (percentage). Between-group comparisons were performed using the Student's t-test or Mann-Whitney U-test for continuous variables, and the chi-square test or Fisher's exact test for categorical variables, as appropriate.

We used maximally selected log-rank statistics from the Maxstat $\mathrm{R}$ package to determine the optimal cut-off value of postoperative weight change (\%) predicting graft failure after LDLT ${ }^{26,27}$. This package tests all possible cutpoints to identify the cutpoint where the maximum of the log-rank statistics is achieved. All patients were classified into 2 groups according to the cutpoint that best discriminates the patients for overall graft survival and failure. The association between postoperative weight change and clinical outcomes after LDLT was evaluated using Cox or logistic regression models. Variables with $P$-values $<0.1$ in univariate analyses were entered into multivariate analyses. Multivariate Cox regression analysis was used to assess the factors associated with graft failure and overall mortality. Multiple logistic regression analysis was used to identify the factors associated with EAD. A backward elimination process was used to develop the final multivariate model, and adjusted hazard ratios (aHRs) or adjusted odds ratios (aORs) with 95\% confidence intervals (CIs) were calculated. The cumulative graft and patient survival rates were calculated using the Kaplan-Meier method, and log-rank tests were used to evaluate the differences between groups.

In addition, the potential nonlinear relationship between postoperative weight change and risk of graft failure was assessed in a multivariate Cox regression model, in which postoperative weight change was modelled with 


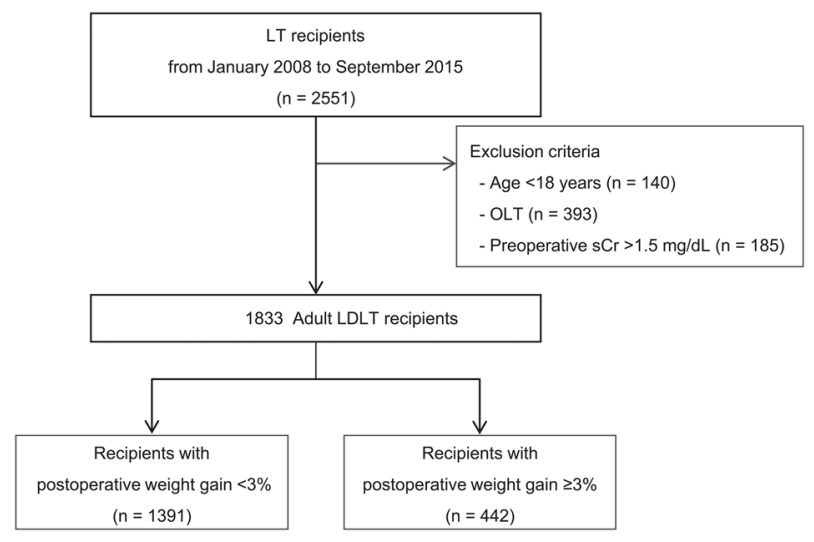

Figure 1. Flowchart of the study population. Abbreviations: LT, liver transplantation; OLT, orthotopic liver transplantation; sCr, serum creatinine; LDLT, living donor liver transplantation.

restricted cubic splines with 5 knots $\left(5^{\text {th }}, 27.5^{\text {th }}, 50^{\text {th }}, 72.5^{\text {th }} \text {, and } 95^{\text {th }} \text { percentiles, as suggested by Harrell }\right)^{28}$; fully aHRs with $95 \%$ CIs were plotted against postoperative weight change.

All reported $P$-values are two-sided, and $P$-values $<0.05$ were considered statistically significant. SPSS 22.0 (SPSS Inc., Chicago, IL, USA) and R 3.5.1 (R Foundation for Statistical Computing, Vienna, Austria) were used for data manipulation and statistical analyses.

\section{Results}

A total of 1833 adult LDLT recipients were included in the final analysis (Fig. 1), and the median postoperative follow-up time was 5.1 years (IQR, 3.4-7.0). The incidence of graft failure was $7.7 \%(\mathrm{n}=142)$. The incidence of EAD was $10.4 \%(n=190)$, and the overall mortality was $6.5 \%(n=119)$. By using the cut-off value determined by the maxstat R package, the 1833 patients were divided into 2 groups based on the amount of postoperative weight gain $(<3 \%$ group $[n=1391]$ and $\geq 3 \%$ group $[n=442])$.

Table 1 shows the baseline characteristics and intraoperative data of the 2 groups. The postoperative weight gain $\geq 3 \%$ group were older, more likely to be female, and had been more frequently diagnosed with diabetes mellitus or hepatic encephalopathy than the postoperative weight gain $<3 \%$ group. In addition, the $\geq 3 \%$ group had larger volumes of crystalloid and albumin infusion, higher amounts of pRBC and FFP transfusion, higher urine output, less intraoperative vasopressor use, and longer warm ischaemic and operation time than the $<3 \%$ group.

Graft failure. The incidence of graft failure was $6.2 \%(n=86)$ in the $<3 \%$ group and $12.7 \%(n=56)$ in the $\geq 3 \%$ group $(P<0.001)$. Postoperative weight gain $\geq 3 \%$ was associated with a significantly higher rate of graft failure (HR, 2.047; 95\% CI, 1.462-2.867; $P<0.001)$ in the univariate Cox proportional hazards model. Other variables related to graft failure are presented in Table 2 . After adjusting for multiple confounders, postoperative weight gain $\geq 3 \%$ was still associated with a higher incidence of graft failure (aHR, 1.763; 95\% CI, 1.248-2.490; $P=0.001)$. Kaplan-Meier survival analysis revealed that the $\geq 3 \%$ group had a significantly higher incidence of graft failure than the $<3 \%$ group (log-rank test, $P<0.001$ ) (Fig. $2 \mathrm{a}$ ).

In addition, we analysed the nonlinear relationship between postoperative weight change (\%) and the risk of graft failure by entering postoperative weight change modelled with restricted cubic splines in a multivariate Cox regression model. This model was adjusted for all variables in Table 2 . Variables with $P$-values $<0.1$ in univariate Cox analysis were selected and included in the final model, which is shown in Fig. 3. After adjusting for confounding factors, LT recipients with postoperative weight gain had higher likelihood of graft failure than those with postoperative weight loss. In addition, postoperative weight change (\%) was entered as a continuous variable in a multivariate Cox model for graft failure, which was adjusted for all variables in Table 2 . The aHR for each $1 \%$ increase in postoperative weight was 1.045 (95\% CI, 1.009-1.082; $P=0.015)$.

Other adverse outcomes. Multiple logistic regression analysis revealed that postoperative weight gain $\geq 3 \%$ was associated with EAD (aOR, 1.553; 95\% CI, 1.024-2.356; $P=0.038$ ) (Table 3). Multivariate Cox proportional regression analysis revealed that postoperative weight gain $\geq 3 \%$ was associated with overall mortality (aHR, 1.731; 95\% CI, 1.182-2.535; $P=0.005$ ). Kaplan-Meier survival analysis revealed that the $\geq 3 \%$ group had a significantly higher overall mortality rate than the $<3 \%$ group (log-rank test, $P<0.001$ ) (Fig. $2 \mathrm{~b}$ ). The $\geq 3 \%$ group had significantly longer ICU stay (median 3.0 days [IQR, 2.0-6.0] versus 3.0 days $[2.0-5.0] ; P<0.001$ ) and hospital stay (34.0 days [27.0-51.0] versus 31.0 days [25.0-43.0]; $P<0.001)$ than the $<3 \%$ group. In addition, we tested our hypothesis with postoperative weight gain cut-off value of 5\% (Supplementary Tables S1, S2 and Fig. S1).

There was no difference in the pulmonary complication rates such as pulmonary edema (222 [16.0\%] vs. 66 [14.9\%], $P=0.658)$, pneumonia $(94[6.8 \%]$ vs. $23[5.2 \%], P=0.292)$, and acute respiratory distress syndrome ( $1[0.1 \%]$ vs. $2[0.5 \%], P=0.294)$ between the postoperative weight gain $<3 \%$ and $\geq 3 \%$ groups. In addition, no difference was found in the incidence of AKI according to the KDIGO criteria (990 [71.3\%] vs. 314 [71.0\%], $P=0.956)$ between the two groups. 


\begin{tabular}{|c|c|c|c|c|}
\hline & $\begin{array}{l}\text { Total } \\
(\mathrm{N}=1833)\end{array}$ & $\begin{array}{l}\text { Weight gain <3\% } \\
(\mathrm{n}=1391)\end{array}$ & $\begin{array}{l}\text { Weight gain } \geq 3 \% \\
(\mathrm{n}=442)\end{array}$ & $P$-value \\
\hline \multicolumn{5}{|l|}{ Demographic } \\
\hline Age (years) & $53.0(49.0-57.0)$ & $53.0(48.0-57.0)$ & $54.0(49.0-58.0)$ & 0.004 \\
\hline Sex, male & $1383(75.5 \%)$ & $1081(77.7 \%)$ & $302(68.3 \%)$ & $<0.001$ \\
\hline Body mass index $\left(\mathrm{kg} / \mathrm{m}^{2}\right)$ & $23.8 \pm 3.3$ & $24.0 \pm 3.2$ & $23.0 \pm 3.2$ & $<0.001$ \\
\hline Diabetes mellitus & $385(21.0 \%)$ & $268(19.3 \%)$ & $117(26.5 \%)$ & 0.002 \\
\hline Hypertension & $236(12.9 \%)$ & $173(12.4 \%)$ & $63(14.3 \%)$ & 0.362 \\
\hline Coronary arterial disease & $65(3.5 \%)$ & $44(3.2 \%)$ & $21(4.8 \%)$ & 0.154 \\
\hline \multicolumn{5}{|l|}{ Liver disease } \\
\hline Fulminant hepatic failure & $78(4.3 \%)$ & $57(4.1 \%)$ & $21(4.8 \%)$ & 0.647 \\
\hline Model for end-stage liver disease score & $12.0(9.0-18.0)$ & $12.0(9.0-18.0)$ & $12.0(9.0-18.0)$ & 0.951 \\
\hline Hepatic encephalopathy & $251(13.7 \%)$ & $169(12.1 \%)$ & $82(18.6 \%)$ & 0.001 \\
\hline Ascites & $554(30.2 \%)$ & $466(33.5 \%)$ & $88(19.9 \%)$ & $<0.001$ \\
\hline Spontaneous bacterial peritonitis & $99(5.4 \%)$ & $80(5.8 \%)$ & $19(4.3 \%)$ & 0.291 \\
\hline Combined hepatocellular carcinoma & $927(50.6 \%)$ & $685(49.2 \%)$ & $242(54.8 \%)$ & 0.050 \\
\hline \multicolumn{5}{|l|}{ Laboratory data } \\
\hline Haemoglobin (g/dL) & $11.0 \pm 2.2$ & $11.0 \pm 2.2$ & $11.1 \pm 2.1$ & 0.553 \\
\hline Prothrombin time (INR) & $1.4(1.2-1.7)$ & $1.4(1.2-1.7)$ & $1.4(1.2-1.6)$ & 0.923 \\
\hline Total bilirubin (mg/dL) & $1.9(1.1-4.5)$ & $1.9(1.1-4.5)$ & $1.9(1.2-4.2)$ & 0.717 \\
\hline Creatinine (mg/dL) & $0.8 \pm 0.2$ & $0.8 \pm 0.2$ & $0.8 \pm 0.2$ & 0.974 \\
\hline Brain natriuretic peptide $(\mathrm{pg} / \mathrm{mL})$ & $40.0(19.0-84.0)$ & $42.0(20.0-85.0)$ & $34.0(16.0-83.0)$ & 0.005 \\
\hline \multicolumn{5}{|l|}{ Donor variables } \\
\hline Donor age (years) & $26.0(21.0-32.0)$ & $26.0(21.0-32.0)$ & $27.0(23.0-32.0)$ & 0.001 \\
\hline Donor sex, male & $1319(72.0 \%)$ & $1021(73.4 \%)$ & $298(67.4 \%)$ & 0.017 \\
\hline Donor body mass index $\left(\mathrm{kg} / \mathrm{m}^{2}\right)$ & $22.9 \pm 2.9$ & $22.9 \pm 2.9$ & $23.1 \pm 2.9$ & 0.201 \\
\hline Total fatty change (\%) & $1.0(0.0-5.0)$ & $1.0(0.0-5.0)$ & $2.0(0.0-5.0)$ & 0.199 \\
\hline Macro fatty change (\%) & $1.0(0.0-3.0)$ & $1.0(0.0-3.0)$ & $1.0(0.0-3.0)$ & 0.295 \\
\hline \multicolumn{5}{|l|}{ Intraoperative data } \\
\hline Graft-to-recipient weight ratio & $1.1 \pm 0.3$ & $1.1 \pm 0.2$ & $1.2 \pm 0.3$ & 0.003 \\
\hline Postreperfusion syndrome & $714(39.0 \%)$ & $542(39.0 \%)$ & $172(38.9 \%)$ & 1.000 \\
\hline Cold ischaemic time (min) & $81.0(66.0-98.0)$ & $81.0(67.0-98.0)$ & $82.0(65.0-100.0)$ & 0.872 \\
\hline Warm ischaemic time (min) & $40.0(33.0-50.0)$ & $40.0(32.0-48.0)$ & $43.0(35.0-57.0)$ & $<0.001$ \\
\hline Operation time $(\mathrm{min})$ & $840.0(760.0-930.0)$ & $822.0(750.0-905.0)$ & $886.5(805.0-975.0)$ & $<0.001$ \\
\hline \multicolumn{5}{|l|}{ Fluid balance } \\
\hline Crystalloid (L) & $6.3(5.0-7.9)$ & $6.0(4.8-7.2)$ & $7.7(6.0-10.3)$ & $<0.001$ \\
\hline Albumin (L) & $3.0(2.3-4.0)$ & $3.0(2.0-3.8)$ & $3.3(2.5-5.0)$ & $<0.001$ \\
\hline Packed red blood cell transfusion (units) & $6.0(2.0-12.0)$ & $6.0(2.0-11.0)$ & $9.0(4.0-18.0)$ & $<0.001$ \\
\hline Fresh frozen plasma transfusion (units) & $7.0(2.0-14.0)$ & $6.0(2.0-12.0)$ & $10.0(5.0-20.0)$ & $<0.001$ \\
\hline Urine output (L) & $1.5(1.0-2.0)$ & $1.4(1.0-1.9)$ & $1.6(1.2-2.4)$ & $<0.001$ \\
\hline Epinephrine use & $687(37.5 \%)$ & $521(37.5 \%)$ & $166(37.6 \%)$ & 1.000 \\
\hline Vasopressor use & $1083(59.1 \%)$ & $848(61.0 \%)$ & $235(53.2 \%)$ & 0.004 \\
\hline
\end{tabular}

Table 1. Baseline characteristics and intraoperative data of living donor liver transplantation recipients and donors. INR, international normalized ratio.

Postoperative inflammatory markers. The mean CRP and NLR levels are presented consecutively from 1 day before surgery to POD 7 (Fig. 4). The mean CRP levels were significantly higher in the $\geq 3 \%$ group than in the $<3 \%$ group from POD 2 onwards (Fig. 4a). The mean NLR values were higher in the $\geq 3 \%$ group than in the $<3 \%$ group starting from POD 4, and statistical significance was achieved from POD 6 onwards (Fig. 4b).

\section{Discussion}

In this retrospective study of 1833 LDLT recipients, we found that early postoperative weight gain was associated with adverse postoperative outcomes. Higher postoperative weight gain was associated with inferior graft survival in LDLT recipients even after adjusting for previously identified risk factors. In addition, recipients with postoperative weight gain $\geq 3 \%$ (as opposed to weight gain $<3 \%$ ) had significantly higher risks of graft failure, EAD, and mortality before and after multivariable adjustment.

Although fluid therapy plays an important role in perioperative care, the optimal approach for fluid administration in patients undergoing major surgery is still controversial ${ }^{29-32}$. Dehydration can lead to dysfunction of vital organs, and adequate fluid administration is essential for maintaining optimal cardiac output, tissue perfusion, and oxygen delivery ${ }^{33,34}$. However, FO has also been reported to be detrimental by affecting multiple organ 


\begin{tabular}{|c|c|c|c|c|c|c|c|c|}
\hline \multirow[b]{3}{*}{ Age (years) } & \multicolumn{4}{|c|}{ Unadjusted } & \multicolumn{4}{|c|}{ Multivariable adjusted $^{\mathrm{a}}$} \\
\hline & \multirow{2}{*}{$\begin{array}{l}\text { HR } \\
1.034\end{array}$} & \multicolumn{2}{|c|}{$95 \% \mathrm{CI}$} & \multirow{2}{*}{\begin{tabular}{|l|}
$P$-value \\
0.003 \\
\end{tabular}} & \multirow{2}{*}{\begin{tabular}{|l|} 
HR \\
1.028 \\
\end{tabular}} & \multicolumn{2}{|c|}{$95 \% \mathrm{CI}$} & \multirow{2}{*}{\begin{tabular}{|l|}
$\boldsymbol{P}$-value \\
0.013 \\
\end{tabular}} \\
\hline & & 1.011 & 1.058 & & & 1.006 & 1.050 & \\
\hline Sex, male & 0.641 & 0.452 & 0.909 & 0.013 & & & & \\
\hline Body mass index $\left(\mathrm{kg} / \mathrm{m}^{2}\right)$ & 1.008 & 0.958 & 1.061 & 0.752 & & & & \\
\hline Diabetes mellitus & 1.325 & 0.911 & 1.927 & 0.142 & & & & \\
\hline Hypertension & 0.718 & 0.414 & 1.247 & 0.239 & & & & \\
\hline Coronary arterial disease & 0.591 & 0.188 & 1.854 & 0.367 & & & & \\
\hline Fulminant hepatic failure & 2.242 & 1.241 & 4.050 & 0.007 & 2.526 & 1.344 & 4.744 & 0.004 \\
\hline Model for end-stage liver disease score & 1.037 & 1.017 & 1.057 & $<0.001$ & & & & \\
\hline Hepatic encephalopathy & 1.844 & 1.238 & 2.746 & 0.003 & & & & \\
\hline Ascites & 1.397 & 0.994 & 1.963 & 0.054 & & & & \\
\hline Spontaneous bacterial peritonitis & 1.343 & 0.706 & 2.554 & 0.369 & & & & \\
\hline Combined hepatocellular carcinoma & 0.931 & 0.670 & 1.293 & 0.669 & & & & \\
\hline Brain natriuretic peptide $(\mathrm{pg} / \mathrm{mL})$ & 1.001 & 1.001 & 1.002 & $<0.001$ & 1.001 & 1.001 & 1.002 & $<0.001$ \\
\hline Donor age (years) & 1.029 & 1.011 & 1.049 & 0.002 & 1.021 & 1.001 & 1.042 & 0.041 \\
\hline Donor sex, male & 0.997 & 0.692 & 1.438 & 0.989 & & & & \\
\hline Donor body mass index $\left(\mathrm{kg} / \mathrm{m}^{2}\right)$ & 1.042 & 0.984 & 1.102 & 0.156 & & & & \\
\hline Total fatty change (\%) & 1.019 & 0.995 & 1.043 & 0.119 & & & & \\
\hline Macro fatty change (\%) & 1.024 & 0.990 & 1.059 & 0.172 & & & & \\
\hline Graft-to-recipient weight ratio & 1.650 & 0.884 & 3.079 & 0.116 & & & & \\
\hline Postreperfusion syndrome & 1.587 & 1.142 & 2.206 & 0.006 & & & & \\
\hline Cold ischaemic time (min) & 1.000 & 0.997 & 1.003 & 0.973 & & & & \\
\hline Warm ischaemic time (min) & 1.001 & 0.997 & 1.005 & 0.547 & & & & \\
\hline Operation time (h) & 1.082 & 1.014 & 1.154 & 0.017 & & & & \\
\hline Packed red blood cell transfusion (units) & 1.019 & 1.012 & 1.026 & $<0.001$ & 1.015 & 1.007 & 1.023 & $<0.001$ \\
\hline Epinephrine use & 1.585 & 1.141 & 2.204 & 0.006 & & & & \\
\hline Vasopressor use & 1.088 & 0.777 & 1.523 & 0.623 & & & & \\
\hline Postoperative weight gain $\geq 3 \%$ & 2.047 & 1.462 & 2.867 & $<0.001$ & 1.763 & 1.248 & 2.490 & 0.001 \\
\hline
\end{tabular}

Table 2. Cox proportional hazards regression analysis of factors associated with graft failure in living donor liver transplantation recipients. HR, hazard ratio; CI, confidence interval. a Adjusted for all variables in the table.

systems ${ }^{13,14}$, resulting in postoperative complications, prolonged hospital stay, and increased mortality in critically ill patients or those undergoing major abdominal surgery ${ }^{1-6}$. Our findings suggest that even a mild degree of FO may be harmful for graft or patient survival in LDLT, making it prudent to avoid postoperative weight gain $\geq 3 \%$ in these patients.

In LT recipients, only a few studies have addressed the association between fluid administration and graft or patient survival ${ }^{8,9}$. In a previous study, the total volume of intraoperative fluid administration was found to be associated with adverse outcomes (either in-hospital death or prolonged postoperative hospitalization [ $>14$ days] associated with morbidity) in LT recipients ${ }^{8}$. After adjusting for age, liver disease severity, intraoperative transfusion volume, inotropic use, and operation time, the volume of intraoperative fluid administration was still independently associated with adverse outcomes. In another study of 1197 LT recipients, a negative correlation was observed between ICU readmission and graft or patient survival ${ }^{9}$. In the subgroup analysis of 53 LT recipients in that study, intravascular FO was suspected to be a significant factor for decompensation leading to ICU readmission in the recipients. Our study is in line with the above studies that addressed the deleterious effects of FO on post-LT outcomes. Moreover, our findings further identified the direct relevance of FO to post-LT graft failure in LDLT recipients.

FO may be causally linked to the development of graft failure in LT recipients. Perioperative acute hypervolaemia may destroy the endothelial surface layer along with the inevitable damage caused by surgical trauma (such as mechanical stress and ischaemia-reperfusion injury), leading to pathologic shifts of fluid and protein towards the interstitium ${ }^{15}$. This is reflected by postoperative weight gain, an indirect measure for the interstitial fluid shift, which leads to bacterial translocation, elevated endotoxin levels, and systemic inflammation ${ }^{15,16}$. Furthermore, pathologic inflammation and endothelial dysfunction are known to promote angiogenesis, fibrogenesis, cirrhosis, and increased hepatic resistance, ultimately resulting in portal hypertension and decreased effective hepatocyte perfusion with the risk of liver failure ${ }^{18-20,35}$. Specifically, CRP has been reported to be associated with extracellular volume expansion ${ }^{36}$ and to have a direct role in endothelial activation and atherosclerosis forma$\operatorname{tion}^{37,38}$. Elevated levels of CRP (preoperatively, early postoperatively, or $>1$ year after transplantation) have been found to be associated with inferior graft survival rates; increased intimal thickening and stenosis after arterial allograft transplantation ${ }^{39}$, higher risk of EAD and mortality after $\mathrm{LT}^{40}$, higher risk of coronary artery disease progression and graft failure after heart transplantation ${ }^{38}$, and rapid deterioration of graft function after renal transplantation ${ }^{41}$. 


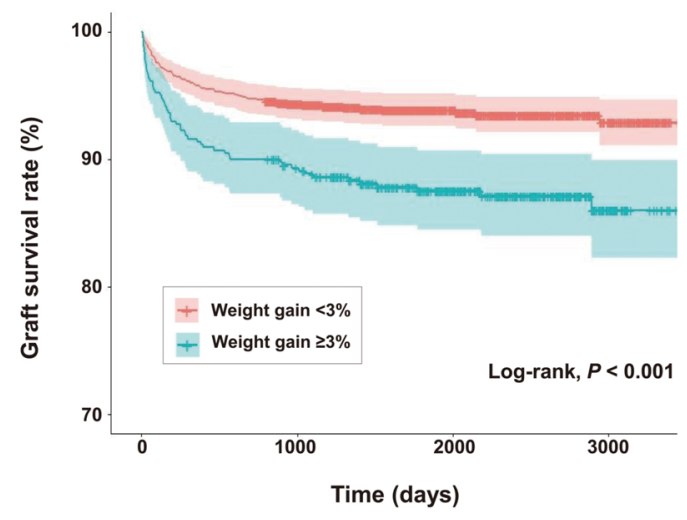

Number at risk

$\begin{array}{rrrr}+\quad 1391 & 1153 & 552 & 139 \\ +\quad 442 & 384 & 250\end{array}$

a

442

384

250

45

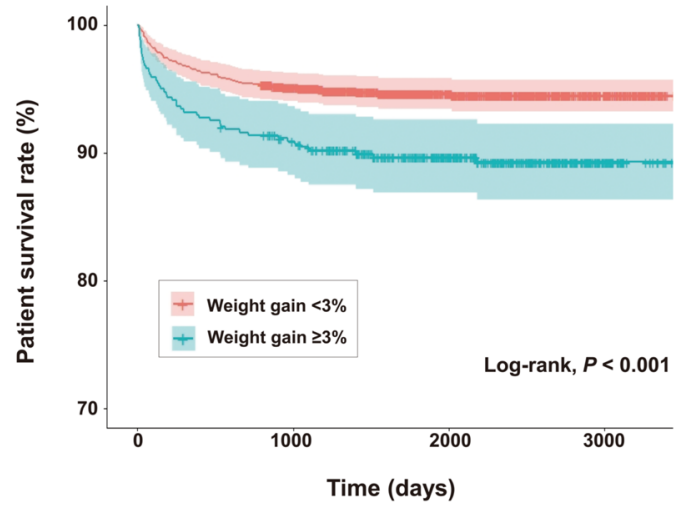

Number at risk

$\begin{array}{rrrrr}+1391 & 1159 & 553 & 140 \\ \text { b } & +\quad 442 & 390 & 256 & 46\end{array}$

Figure 2. (a) Graft and (b) patient survival rates stratified by postoperative weight gain $(<3 \%$ or $\geq 3 \%)$ in living donor liver transplantation recipients. Survival curves are provided with $95 \%$ confidence intervals.

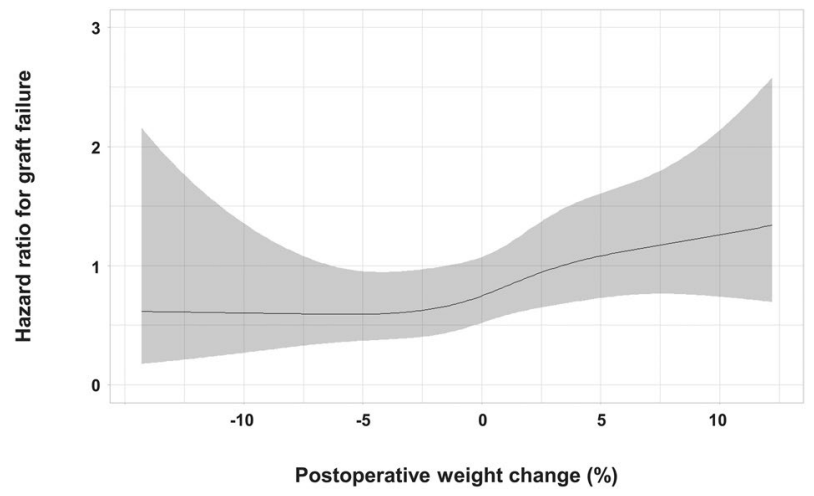

Figure 3. Postoperative weight change and risk of graft failure in living donor liver transplantation recipients. The solid black line represents the adjusted hazard ratio for the association between postoperative weight change and graft failure, and grey shading represents the $95 \%$ confidence interval of the estimate. Postoperative weight change was modelled with restricted cubic splines with 5 knots $\left(5^{\text {th }}, 27.5^{\text {th }}, 50^{\text {th }}, 72.5^{\text {th }}\right.$, and $95^{\text {th }}$ percentiles) in a multivariate Cox model, which was adjusted for all variables in Table 2. 


\begin{tabular}{|c|c|c|c|c|c|c|c|c|c|c|}
\hline \multirow[b]{3}{*}{$\begin{array}{l}\text { Early allograft } \\
\text { dysfunction }\end{array}$} & \multirow[b]{3}{*}{$\begin{array}{l}\text { Weight gain } \geq 3 \% \\
\text { Weight gain }<3 \%\end{array}$} & \multirow{4}{*}{\begin{tabular}{|l|} 
Event/n \\
$60 / 442$ \\
$130 / 1391$ \\
Event/n \\
\end{tabular}} & \multicolumn{4}{|c|}{ Unadjusted } & \multicolumn{4}{|c|}{ Multivariable adjusted $^{\mathrm{a}}$} \\
\hline & & & \multirow{2}{*}{\begin{tabular}{|l|} 
OR \\
1.524 \\
1
\end{tabular}} & \multicolumn{2}{|c|}{$95 \% \mathrm{CI}$} & \multirow{3}{*}{\begin{tabular}{|l|}
$\boldsymbol{P}$-value \\
0.012 \\
$\boldsymbol{P}$-value \\
\end{tabular}} & \multirow{2}{*}{\begin{tabular}{|l|} 
OR \\
1.553 \\
1
\end{tabular}} & \multicolumn{2}{|c|}{ 95\% CI } & \multirow{2}{*}{\begin{tabular}{|l|}
$\boldsymbol{P}$-value \\
0.038 \\
\end{tabular}} \\
\hline & & & & 1.099 & 2.112 & & & 1.024 & 2.356 & \\
\hline & & & HR & $95 \% \mathrm{C}$ & & & HR & $95 \% \mathrm{C}$ & & $P$-value \\
\hline Graft failure & $\begin{array}{l}\text { Weight gain } \geq 3 \% \\
\text { Weight gain }<3 \%\end{array}$ & \begin{tabular}{|l|}
$56 / 442$ \\
$86 / 1391$
\end{tabular} & $\begin{array}{l}2.047 \\
1\end{array}$ & 1.462 & 2.867 & $<0.001$ & $\begin{array}{l}1.763 \\
1\end{array}$ & 1.248 & 2.490 & 0.001 \\
\hline Overall mortality & $\begin{array}{l}\text { Weight gain } \geq 3 \% \\
\text { Weight gain }<3 \%\end{array}$ & \begin{tabular}{|l|}
$46 / 442$ \\
$73 / 1391$
\end{tabular} & $\begin{array}{l}1.990 \\
1\end{array}$ & 1.376 & 2.879 & $<0.001$ & $\begin{array}{l}1.731 \\
1\end{array}$ & 1.182 & 2.535 & 0.005 \\
\hline
\end{tabular}

Table 3. Predictive value of postoperative weight gain $\geq 3 \%$ for clinical outcomes in living donor liver transplantation recipients. OR, odds ratio; $\mathrm{HR}$, hazard ratio; $\mathrm{CI}$, confidence interval. ${ }^{a}$ Adjusted for all variables in Table 2.
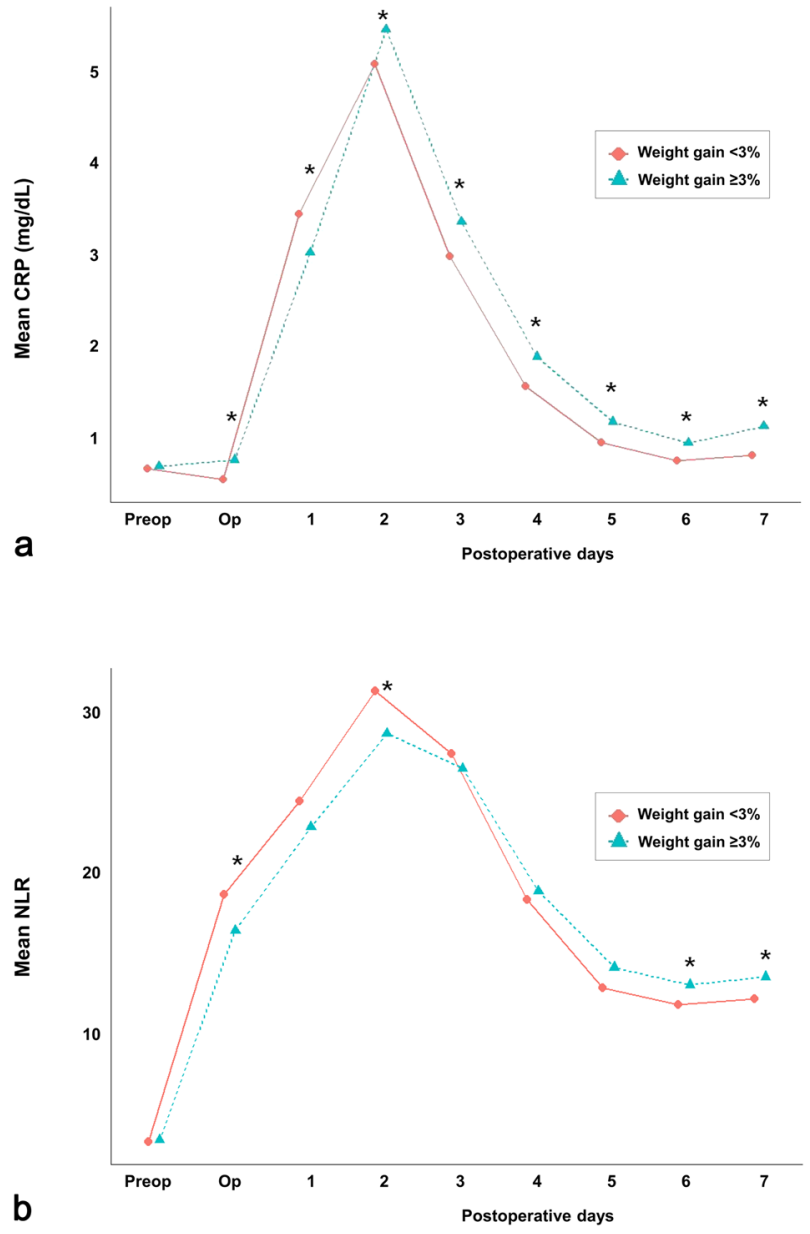

Figure 4. Sequential changes in mean (a) CRP and (b) NLR values in living donor liver transplantation recipients, $* P<0.05$. Abbreviations: CRP, C-reactive protein; NLR, neutrophil-to-lymphocyte ratio; Preop, preoperative day; Op, operation day.

In this study, in agreement with the above studies that elaborated the association between FO, inflammation, and organ or graft dysfunction ${ }^{15,16,18-20,35-41}$, the postoperative weight gain $\geq 3 \%$ group had significantly greater mean CRP levels from POD 2 and significantly higher risks of EAD, graft failure, and mortality than the postoperative weight gain $<3 \%$ group. Previous studies have reported that patients who gain at least $2.5-3 \mathrm{~kg}$ of body weight after major abdominal surgery have poorer clinical outcomes than those who maintained a state of zero fluid balance ${ }^{1-3,31}$. Our findings also suggest that even a mild postoperative weight gain could be associated with inferior graft and patient survival after LT. However, considering the potential harmful effects of volume depletion on other organs, a negative fluid-balance might also have adverse effects in LT recipients. Therefore, we presume that careful fluid administration (in terms of type, volume, and timing) would be necessary in LDLT recipients for maintaining normovolaemia, to ensure adequate perfusion of multiple organs and at the same time to avoid postoperative weight gain ${ }^{15,42}$. 
Patient or graft survival after LT has been reported to be inversely related to older donor age, older recipient age, acute liver failure, and higher RBC transfusion requirements ${ }^{11,12,43,44}$. Preoperative BNP level has also been found to be an independent predictor of post-LT ICU mortality ${ }^{45}$. Therefore, our findings of the factors associated with graft failure are in line with previous findings.

This study has some limitations. Because of the retrospective design, the effects of confounding factors on post-LT graft failure could not be completely ruled out. Further, although a causal relationship between postoperative weight gain and graft failure could be inferred based on the physiology or previous studies, it could not be confirmed. Additionally, because the data were collected from a single centre, idiosyncrasies of local surgical and perioperative management of patients may have affected clinical outcomes. Therefore, large-scale multicentre prospective studies are needed to confirm the causal relationship between postoperative weight gain and graft failure in LDLT recipients.

In conclusion, the results of this large observational study suggest that early postoperative weight gain may be associated with systemic inflammation and deterioration of graft function in LDLT recipients. Additionally, postoperative weight gain $\geq 3 \%$ (as opposed to weight gain $<3 \%$ ) was associated with significantly higher risks of EAD and mortality after LDLT.

\section{Data availability}

The datasets generated during and/or analysed during the current study are available from the corresponding author on reasonable request.

Received: 5 July 2019; Accepted: 13 December 2019;

Published online: 27 December 2019

\section{References}

1. Nisanevich, V. et al. Effect of intraoperative fluid management on outcome after intraabdominal surgery. Anesthesiology 103, 25-32 (2005).

2. Brandstrup, B. et al. Effects of intravenous fluid restriction on postoperative complications: comparison of two perioperative fluid regimens: a randomized assessor-blinded multicenter trial. Ann. Surg. 238, 641-648 (2003).

3. Lobo, D. N. et al. Effect of salt and water balance on recovery of gastrointestinal function after elective colonic resection: a randomised controlled trial. Lancet 359, 1812-1818 (2002).

4. Bouchard, J. et al. Fluid accumulation, survival and recovery of kidney function in critically ill patients with acute kidney injury. Kidney Int. 76, 422-427 (2009).

5. The National Heart, Lung, and Blood Institute Acute Respiratory Distress Syndrome (ARDS) Clinical Trials Network. Comparison of two fluid-management strategies in acute lung injury. N. Engl. J. Med. 354, 2564-2575 (2006).

6. Vidal, M. G. et al. Incidence and clinical effects of intra-abdominal hypertension in critically ill patients. Crit. Care Med. 36, 1823-1831 (2008).

7. Dekker, M. J. et al. Impact of fluid status and inflammation and their interaction on survival: a study in an international hemodialysis patient cohort. Kidney Int. 91, 1214-1223 (2017).

8. Bennett-Guerrero, E. et al. Preoperative and intraoperative predictors of postoperative morbidity, poor graft function, and early rejection in 190 patients undergoing liver transplantation. Arch. Surg. 136, 1177-1183 (2001).

9. Levy, M. F. et al. Readmission to the intensive care unit after liver transplantation. Crit. Care Med. 29, 18-24 (2001).

10. Jiang, G. Q., Peng, M. H. \& Yang, D. H. Effect of perioperative fluid therapy on early phase prognosis after liver transplantation. HBPD Int 7, 367-372 (2008).

11. Habib, S. et al. MELD and prediction of post-liver transplantation survival. Liver Transpl. 12, 440-447 (2006)

12. Moore, D. E. et al. Impact of donor, technical, and recipient risk factors on survival and quality of life after liver transplantation. Arch. Surg. 140, 273-277 (2005).

13. Holte, K., Sharrock, N. E. \& Kehlet, H. Pathophysiology and clinical implications of perioperative fluid excess. Br. J. Anaesth. 89, 622-632 (2002).

14. Prowle, J. R., Echeverri, J. E., Ligabo, E. V., Ronco, C. \& Bellomo, R. Fluid balance and acute kidney injury. Nat. Rev. Nephrol. 6 , 107-115 (2010).

15. Chappell, D., Jacob, M., Hofmann-Kiefer, K., Conzen, P. \& Rehm, M. A rational approach to perioperative fluid management. Anesthesiology 109, 723-740 (2008).

16. Reyes-Bahamonde, J., Raimann, J. G., Thijssen, S., Levin, N. W. \& Kotanko, P. Fluid overload and inflammation: a vicious cycle. Semin. Dial. 26, 31-35 (2013).

17. Bernardi, M., Moreau, R., Angeli, P., Schnabl, B. \& Arroyo, V. Mechanisms of decompensation and organ failure in cirrhosis: from peripheral arterial vasodilation to systemic inflammation hypothesis. J. Hepatol. 63, 1272-1284 (2015).

18. Fernandez, M. et al. Angiogenesis in liver disease. J. Hepatol. 50, 604-620 (2009).

19. Herrmann, J. et al. Transcapsular arterial neovascularization after liver transplantation in pediatric patients indicates transplant failure. Radiology 261, 566-572 (2011).

20. Robinson, M. W., Harmon, C. \& O’Farrelly, C. Liver immunology and its role in inflammation and homeostasis. Cell. Mol. Immunol. 13, 267-276 (2016).

21. Kim, Y. K. et al. Effect of right ventricular dysfunction on dynamic preload indices to predict a decrease in cardiac output after inferior vena cava clamping during liver transplantation. Transplant. Proc. 42, 2585-2589 (2010).

22. Feldheiser, A. et al. Enhanced Recovery After Surgery (ERAS) for gastrointestinal surgery, part 2: consensus statement for anaesthesia practice. Acta Anaesthesiol. Scand. 60, 289-334 (2016).

23. Ljungqvist, O., Scott, M. \& Fearon, K. C. Enhanced Recovery After Surgery: A Review. JAMA Surg. 152, 292-298 (2017).

24. Olthoff, K. M. et al. Liver regeneration after living donor transplantation: adult-to-adult living donor liver transplantation cohort study. Liver Transpl. 21, 79-88 (2015).

25. Kidney Disease: Improving Global Outcomes (KDIGO) Acute Kidney Injury Work Group. Section 2: AKI Definition. Kidney Int Suppl. 2, 19-36 (2012).

26. Hothorn, T. \& Lausen, B. On the exact distribution of maximally selected rank statistics. Comput. Stat. Data Anal. 43, 121-137 (2003).

27. Lausen, B. \& Schumacher, M. Maximally Selected Rank Statistics. Biometrics 48(1), 73-85 (1992).

28. Harrell, F. E. Jr. Regression modeling strategies: with applications to linear models, logistic regression, and survival analysis. 568 (Springer, 2001). 
29. Corcoran, T., Rhodes, J. E., Clarke, S., Myles, P. S. \& Ho, K. M. Perioperative fluid management strategies in major surgery: a stratified meta-analysis. Anesth. Analg. 114, 640-651 (2012).

30. Myles, P. S. et al. Restrictive versus liberal fluid therapy for major abdominal surgery. N. Engl. J. Med. 378, 2263-2274 (2018).

31. Varadhan, K. K. \& Lobo, D. N. A meta-analysis of randomised controlled trials of intravenous fluid therapy in major elective open abdominal surgery: getting the balance right. Proc. Nutr. Soc. 69, 488-498 (2010).

32. Holte, K. \& Kehlet, H. Fluid therapy and surgical outcomes in elective surgery: a need for reassessment in fast-track surgery. J. Am. Coll. Surg. 202, 971-989 (2006).

33. Sear, J. W. Kidney dysfunction in the postoperative period. Br. J. Anaesth. 95, 20-32 (2005).

34. Arkilic, C. F. et al. Supplemental perioperative fluid administration increases tissue oxygen pressure. Surgery 133, 49-55 (2003).

35. Poisson, J. et al. Liver sinusoidal endothelial cells: physiology and role in liver diseases. J. Hepatol. 66, 212-227 (2017).

36. Konings, C. J. et al. Fluid status in CAPD patients is related to peritoneal transport and residual renal function: evidence from a longitudinal study. Nephrol. Dial. Transplant. 18, 797-803 (2003).

37. Labarrere, C. A. \& Zaloga, G. P. C-reactive protein: from innocent bystander to pivotal mediator of atherosclerosis. Am. J. Med. 117, 499-507 (2004).

38. Labarrere, C. A. et al. C-reactive protein, arterial endothelial activation, and development of transplant coronary artery disease: a prospective study. Lancet 360, 1462-1467 (2002).

39. Fitzgerald, J. T. et al. Occult pretransplantation systemic inflammation and posttransplantation vascular changes in a primate arterial allograft model. Transplantation 78, 367-374 (2004).

40. Oweira, H. et al. Early post-operative acute phase response in patients with early graft dysfunction is predictive of 6-month and 12-month mortality in liver transplant recipients. Hum. Immunol. 77, 952-960 (2016).

41. van Ree, R. M. et al. Elevated levels of C-reactive protein independently predict accelerated deterioration of graft function in renal transplant recipients. Nephrol. Dial. Transplant. 22, 246-253 (2007).

42. Jacob, M., Chappell, D. \& Rehm, M. Clinical update: perioperative fluid management. Lancet 369, 1984-1986 (2007).

43. Burroughs, A. K. et al. 3-month and 12-month mortality after first liver transplant in adults in Europe: predictive models for outcome. Lancet 367, 225-232 (2006).

44. de Boer, M. T. et al. The impact of intraoperative transfusion of platelets and red blood cells on survival after liver transplantation. Anesth. Analg. 106, 32-44 (2008).

45. Toussaint, A. et al. Prognostic value of preoperative brain natriuretic peptide serum levels in liver transplantation. Transplantation 100, 819-824 (2016).

\section{Author contributions}

H.W.J., MD. This author made substantial contribution to the study concept and design; acquisition, analysis, and interpretation of data; drafting of the manuscript; study supervision and revision. J.G.S., MD., PhD. This author made substantial contribution to the study concept and design; acquisition, analysis, and interpretation of data; drafting of the manuscript; study supervision and revision. K.W.J., MD. This author made substantial contribution to the acquisition of data. S.O.K. This author made substantial contribution to the statistical analysis. H.M.K., MD. This author made substantial contribution to the statistical analysis. Y.J.M., MD. This author made substantial contribution to the acquisition of data. I.G.J., MD., PhD. This author made substantial contribution to the acquisition of data. G.S.H., MD., PhD. This author made substantial contribution to the study design and supervision.

\section{Competing interests}

The authors declare no competing interests.

\section{Additional information}

Supplementary information is available for this paper at https://doi.org/10.1038/s41598-019-56543-3.

Correspondence and requests for materials should be addressed to J.-G.S.

Reprints and permissions information is available at www.nature.com/reprints.

Publisher's note Springer Nature remains neutral with regard to jurisdictional claims in published maps and institutional affiliations.

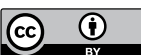

Open Access This article is licensed under a Creative Commons Attribution 4.0 International License, which permits use, sharing, adaptation, distribution and reproduction in any medium or format, as long as you give appropriate credit to the original author(s) and the source, provide a link to the Creative Commons license, and indicate if changes were made. The images or other third party material in this article are included in the article's Creative Commons license, unless indicated otherwise in a credit line to the material. If material is not included in the article's Creative Commons license and your intended use is not permitted by statutory regulation or exceeds the permitted use, you will need to obtain permission directly from the copyright holder. To view a copy of this license, visit http://creativecommons.org/licenses/by/4.0/.

(C) The Author(s) 2019 